\title{
THE DENSITY OF EXTREME POINTS IN COMPLEX POLYNOMIAL APPROXIMATION
}

\author{
ANDRÁS KROÓ AND E. B. SAFF
}

(Communicated by Irwin Kra)

\begin{abstract}
Let $K$ be a compact set in the complex plane having connected and regular complement, and let $f$ be any function continuous on $K$ and analytic in the interior of $K$. For the polynomials $p_{n}^{*}(f)$ of respective degrees at most $n$ of best uniform approximation to $f$ on $K$, we investigate the density of the sets of extreme points
\end{abstract}

$$
A_{n}(f):=\left\{z \in K:\left|f(z)-p_{n}^{*}(f)(z)\right|=\left\|f-p_{n}^{*}(f)\right\|_{K}\right\}
$$

in the boundary of $K$.

1. Introduction. Let $K$ be a compact set in the complex plane $\mathbf{C}$ and consider the space $C(K)$ of complex-valued continuous functions on $K$ endowed with the sup norm

$$
\|f\|_{K}:=\max _{z \in K}|f(z)| \text {. }
$$

For a given $f \in C(K)$ we denote by $p_{n}^{*}(f)$ its best approximant out of $P_{n}$, the set of all algebraic polynomials of degree at most $n(n \in \mathbf{N})$. We let

$$
A_{n}(f):=\left\{z \in K:\left|f(z)-p_{n}^{*}(f)(z)\right|=\left\|f-p_{n}^{*}(f)\right\|_{K}\right\}
$$

and refer to $A_{n}(f)$ as the set of extreme points for $n$th degree polynomial approximation to $f$. The purpose of this paper is to investigate the density of the sets $A_{n}(f)$ in the boundary of $K$.

For the case of best polynomial approximation to a real-valued function $f$ on an interval $K=[a, b]$, Kadec [2] established the uniform distribution (with respect to the Chebyshev measure) of such extreme point sets for a subsequence of integers (depending on the function). Lorentz [4] showed later that, in general, Kadec's result does not hold for all subsequences. Fuchs [1] extended the work of Kadec by studying the limiting distribution of extreme points for real polynomial approximation on a compact set $K \subset \mathbf{R}$.

In [3 and 8] some estimates for the density of extreme points are given for the cases of real approximation on an interval and approximation on the unit disk, respectively. In particular, it is shown in [3] that if $K$ is the closed unit disk and $f \in C(K)$ is analytic in the interior of $K$, then the extreme points for best polynomial approximation to $f$ are dense on $\partial K$ (the boundary of $K$ ). In this note

Received by the editors February 13, 1987.

1980 Mathematics Subject Classification (1985 Revision). Primary 41A50, 30E10.

Key words and phrases. Polynomial approximation, extreme points, Chebyshev polynomials, best approximants.

The research of the second author was supported in part by the National Science Foundation under grant DMS-840 1370. 
we shall extend this result to any compact set $K \subset \mathbf{C}$ whose complement (with respect to the extended plane $\overline{\mathbf{C}}$ ) is connected and regular with respect to the Dirichlet problem (cf. [9, p. 6]). We also provide a Lorentz-type counterexample, showing that the density holds, in general, only for a subsequence of integers.

Main results. We measure the density of a set $A$ in the set $B(A, B \subset \mathbf{C})$ by

$$
\rho(A, B):=\sup _{z \in B} \inf _{\zeta \in A}|z-\varsigma| \text {. }
$$

Our main result concerning the density of the extreme point sets $A_{n}(f)$ defined in (1.1) is the following.

THEOREM 2.1. Let $K \subset \mathbf{C}$ be a compact set with connected and regular complement. If $f \in C(K)$ is analytic in $\operatorname{Int}(K)$, then

$$
\liminf _{n \rightarrow \infty} \rho\left(A_{n}(f), \partial K\right)=0 .
$$

Moreover, there exists an entire function $g$ such that

$$
\limsup _{n \rightarrow \infty} \rho\left(A_{n}(g), \partial K\right)>0 .
$$

REMARK (i). Theorem 2.1 applies, in particular, if $K$ is any continuum (not a single point), since the complement of such a set is simply connected and hence regular.

REMARK (ii). As will be seen from the proof of Theorem 2.1, the relation (2.2) also holds if the set $A_{n}(f)$ is replaced by an extremal signature, which is a discrete subset of $A_{n}(f)$ consisting of at most $2 n+3$ points (see [7] for the precise definition).

REMARK (iii). The methods of Kadec and Fuchs that are used to establish stronger results concerning the distribution of extreme points for real approximation are not applicable in the general setting of Theorem 2.1. Indeed, the previously cited results of these authors are based on the asymptotic behavior of the zeros of the polynomial differences $p_{n}^{*}(f)-p_{n-1}^{*}(f)$. For real approximation such zeros interlace extreme points, but no such relationship is known in the complex setting.

The proof of Theorem 2.1 requires several lemmas, some of which are of independent interest. In what follows, $\operatorname{cap}(B)$ denotes the logarithmic capacity (transfinite diameter) of the set $B \subset \mathbf{C}$. We note that the regularity assumption of Theorem 2.1 implies that $\operatorname{cap}(K)>0$. With $K$ as in Theorem 2.1 we first establish

LEMMA 2.2. Let $z_{0} \in \partial K$. Then $\operatorname{cap}(K \backslash U)<\operatorname{cap}(K)$ for every neighborhood (open disk) $\mathcal{U}$ of $z_{0}$.

PROOF. For a compact set $V \subset \mathbf{C}$, let $g_{V}(z, \infty)$ denote the Green's function with pole at $\infty$ for the unbounded component of $\overline{\mathbf{C}} \backslash V$. Suppose to the contrary that

$$
\operatorname{cap}(K \backslash \mathcal{U})=\operatorname{cap}(K)
$$

for some neighborhood $\mathcal{U}$ of $z_{0}$. Since

$$
\lim _{z \rightarrow \infty}\left[\log |z|-g_{V}(z, \infty)\right]=\log [\operatorname{cap}(V)]
$$

(2.4) implies that

$$
\lim _{z \rightarrow \infty}\left[g_{K}(z, \infty)-g_{K \backslash u}(z, \infty)\right]=0
$$


By the regularity assumption, $g_{K}(z, \infty)$ is continuous on $\overline{\mathbf{C} \backslash K}$ and $g_{K}(z, \infty)=0$ on $\partial K$. Also, $g_{K \backslash u}(z, \infty) \geq 0$ as $z \rightarrow \partial K$, and hence

$$
\limsup _{z \rightarrow \partial K}\left[g_{K}(z, \infty)-g_{K \backslash u}(z, \infty)\right] \leq 0 .
$$

Thus, by the maximum principle for harmonic functions we have $g_{K}(z, \infty)=$ $g_{K \backslash u}(z, \infty)$ for $z \in \mathbf{C} \backslash K$. Consequently, $g_{K}\left(z_{0}, \infty\right)=g_{K \backslash u}\left(z_{0}, \infty\right)$. But $z_{0}$ belongs to the unbounded component of the complement of $K \backslash \mathcal{U}$ and hence $g_{K \backslash u}\left(z_{0}, \infty\right)>0$. On the other hand, $g_{K}\left(z_{0}, \infty\right)=0$, which yields the desired contradiction.

For a compact set $B \subset \mathbf{C}$ we set

$$
e_{n}(B):=\min _{p \in \mathcal{P}_{n-1}}\left\|z^{n}-p(z)\right\|_{B}
$$

Furthermore, let

$$
E_{n}(f):=\inf _{p \in \mathcal{P}_{n}}\|f-p\|_{K}=\left\|f-p_{n}^{*}(f)\right\|_{K}
$$

With the above notation we next establish

LEMMA 2.3. Let $f \in C(K) \backslash P_{n-1}$. Then

$$
\frac{E_{n-1}(f)-E_{n}(f)}{E_{n-1}(f)+E_{n}(f)} \leq \frac{e_{n}\left(A_{n-1}(f)\right)}{e_{n}(K)}, \quad n=1,2, \ldots
$$

PROOF. Write $p_{n}^{*}(f)(z)=a_{n} z^{n}+\cdots, a_{n} \in \mathbf{C}$, and let $T_{n}^{*}(z)=z^{n}+\cdots \in P_{n}$ be the Chebyshev polynomial of degree $n$ for the set $A_{n-1}(f)$; that is,

$$
e_{n}\left(A_{n-1}(f)\right)=\left\|T_{n}^{*}\right\|_{A_{n-1}(f)} .
$$

From properties of extremal signatures, it is known (cf. $[\mathbf{7}$, p. 15]) that

$$
E_{n-1}(f)=\left\|f-p_{n-1}^{*}(f)\right\|_{K}=\inf _{p \in P_{n-1}}\|f-p\|_{A_{n-1}(f)} .
$$

Since $p_{n}^{*}(f)-a_{n} T_{n}^{*} \in P_{n-1}$, we see from (2.10) and (2.9) that

$$
E_{n-1}(f) \leq\left\|f-p_{n}^{*}(f)+a_{n} T_{n}^{*}\right\|_{A_{n-1}(f)} \leq E_{n}(f)+\left|a_{n}\right| e_{n}\left(A_{n-1}(f)\right) .
$$

We may assume that $a_{n} \neq 0$ since otherwise $E_{n}(f)=E_{n-1}(f)$ and (2.8) is trivial. Then we have, by the triangle inequality,

$$
e_{n}(K) \leq\left\|\frac{p_{n}^{*}(f)-p_{n-1}^{*}(f)}{a_{n}}\right\|_{K} \leq \frac{E_{n-1}(f)+E_{n}(f)}{\left|a_{n}\right|} .
$$

Combining (2.12) with (2.11) we get (2.8).

Lemmas 2.2 and 2.3 yield a result of independent interest concerning the density of the extreme points for the Chebyshev polynomials associated with the set $K$. Namely, we have

COROLlARY 2.4. Let $T_{n}(z)=T_{n}(K ; z)=z^{n}+\cdots \in P_{n}$ be the nth degree Chebyshev polynomial for a compact set $K$ that has connected and regular complement. For $n=1,2, \ldots$, set

$$
A_{n}^{*}(K):=\left\{z \in \partial K:\left|T_{n}(z)\right|=\left\|T_{n}\right\|_{K}=e_{n}(K)\right\} .
$$


Then

$$
\lim _{n \rightarrow \infty} \rho\left(A_{n}^{*}(K), \partial K\right)=0 .
$$

ProOF. Assume that (2.14) is false. Then there exists a $\delta>0$ and an increasing sequence $\Lambda$ of positive integers such that $\rho\left(A_{n}^{*}(K), \partial K\right) \geq \delta$ for $n \in \Lambda$. Since $\partial K$ is compact, there exist a $z_{0} \in \partial K$, a neighborhood $\mathcal{U}$ of $z_{0}$, and a subsequence $\Lambda_{0} \subset \Lambda$ such that $A_{n}^{*}(K) \subset K \backslash \mathcal{U}$, for $n \in \Lambda_{0}$. Then, from (2.8) with $f(z)=z^{n}$, we have

$$
1 \leq \frac{e_{n}\left(A_{n}^{*}(K)\right)}{e_{n}(K)} \leq \frac{e_{n}(K \backslash \mathcal{U})}{e_{n}(K)} \quad \text { for } n \in \Lambda_{0} .
$$

On the other hand, it is known (cf. [9, p. 73]) that, for any compact set $B \subset \mathbf{C}$,

$$
\lim _{n \rightarrow \infty}\left[e_{n}(B)\right]^{1 / n}=\operatorname{cap}(B) .
$$

Thus it follows from (2.15) that $\operatorname{cap}(K) \leq \operatorname{cap}(K \backslash \mathcal{U})$, contradicting Lemma 2.2.

We can now prove the first assertion of Theorem 2.1.

PROOF OF (2.2). We may assume that $f$ is not a polynomial since, otherwise, the assertion is trivial. By Mergelyan's theorem, $E_{n}(f) \rightarrow 0$ as $n \rightarrow \infty$, which implies (as observed by Kadec [2]) that $\sum_{n=1}^{\infty} \lambda_{n}=\infty$, where

$$
\lambda_{n}:=\left[E_{n-1}(f)-E_{n}(f)\right] /\left[E_{n-1}(f)+E_{n}(f)\right] .
$$

Hence there exists an increasing sequence $\Lambda$ of positive integers such that

$$
\lambda_{n} \geq 1 / n^{2} \text { for } n \in \Lambda \text {. }
$$

If (2.2) is false, there exist $z_{0} \in \partial K$, a neighborhood $\mathcal{U}$ of $z_{0}$, and a subsequence $\Lambda_{0} \subset \Lambda$ such that $A_{n-1}(f) \subset K \backslash \mathcal{U}$ for $n \in \Lambda_{0}$. Then, by (2.8),

$$
\lambda_{n} \leq e_{n}(K \backslash \mathcal{U}) / e_{n}(K) \text { for } n \in \Lambda_{0} .
$$

But, as in the proof of Corollary 2.4, Lemma 2.2 implies that the right-hand member of (2.19) tends to zero exponentially, contradicting (2.18).

REMARK (iv). It follows from the above proof that if $\Lambda$ is any increasing sequence of integers for which

$$
\lim _{n \rightarrow \infty} \lambda_{n}^{1 / n}=1, \quad n \in \Lambda
$$

then

$$
\lim _{n \rightarrow \infty} \rho\left(A_{n}(f), \partial K\right)=0, \quad n \in \Lambda .
$$

For example, if for all $n \geq n_{0}$, we have $E_{n}(f) \leq\left(1-\varepsilon_{n}\right) E_{n-1}(f)$ with $\log \varepsilon_{n}=o(n)$ $\left(0<\varepsilon_{n}<1\right)$, then $\lim _{n \rightarrow \infty} \rho\left(A_{n}(f), \partial K\right)=0$.

REMARK (v). In some special cases it is possible to give explicit estimates for the quantity $e_{n}(B)$ defined in (2.6). Such estimates together with relation (2.8) allow one to estimate how fast $\rho\left(A_{n}(f), \partial K\right)$ tends to zero on a subsequence, yielding in particular the result of [3].

3. A Lorentz-type counterexample. To establish the second part of Theorem 2.1 we require some auxiliary results. The first is the Bernstein-Walsh lemma. 
LEMMA $3.1[\mathbf{1 0}, \mathrm{P} .77]$. Let $K \subset \mathbf{C}$ be a compact set with $\operatorname{cap}(K)>0$. If $p \in P_{n}$ satisfies $\|p\|_{K} \leq M$, then

$$
|p(z)| \leq M \exp \left(n g_{K}(z, \infty)\right), \quad z \in \Omega
$$

where $\Omega$ is the unbounded component of the complement of $K$.

LEMMA 3.2. Let $K \subset \mathbf{C}$ be compact with $\operatorname{cap}(K)>0$. For a given $z_{0} \in K$, consider a sequence of polynomials

$$
q_{2 n}(z):=\left(z-z_{0}\right)^{n} p_{n}(z), \quad p_{n} \in P_{n}, n=0,1, \ldots,
$$

such that $\left\|q_{2 n}\right\|_{K} \leq 1$ for all $n$. Then there exists a $\delta>0$ such that $q_{2 n}(z)$ tends to zero uniformly on $B_{\delta}:=\left\{z:\left|z-z_{0}\right|<\delta\right\}$.

PROOF. Set $K_{\delta}:=K \backslash B_{\delta}$. It can be easily seen that $\operatorname{cap}\left(K_{\delta}\right)>0$ for $0<\delta \leq \delta_{0}$. Furthermore, we have $\left|\delta_{0}^{n} p_{n}(z)\right| \leq 1$ for all $z \in K_{\delta_{0}}$. Applying now Lemma 3.1, we can find a constant $M_{0}>0$ (independent of $n$ ) such that $\left|p_{n}(z)\right| \leq M_{0}^{n}$ for $z \in \bar{B}_{\delta_{0}}$. Thus, for every $0<\delta \leq \delta_{0}$ and $z \in B_{\delta}$, we have $\left|q_{2 n}(z)\right| \leq \delta^{n} M_{0}^{n}, n=0,1, \ldots$ Finally, choosing $\delta<1 / M_{0}$, we can ensure that $q_{2 n}(z)$ tends uniformly to 0 on $B_{\delta}$.

Our next statement is a straightforward consequence of a well-known result of Krein (cf. [6, p. 12]) stating that given an $(n+1)$-dimensional space $U_{n+1}$ and an $n$-dimensional space $V_{n}$ in a (possibly complex) Banach space $X$, there exists a $q \in U_{n+1} \backslash\{0\}$ having 0 as its best approximant out of $V_{n}$.

LEMMA 3.3. Let $K \subset \mathbf{C}$ be compact and $z_{0} \in K$. Then there exists a polynomial $q_{2 n}(z)$ ( $\left.\not \equiv 0\right)$ of the form (3.2) such that 0 is its best uniform approximant on $K$ out of $P_{n-1}$.

Finally, we need a lemma on the Lipschitz continuity of the best approximation operator, which follows from the complex version of the strong uniqueness theorem due to Newman and Shapiro [5].

LeMma 3.4. Let $K \subset \mathbf{C}$ be compact and $f \in C(K)$. Then for every $g \in C(K)$ satisfying $\|f-g\|_{K} \leq 1$,

$$
\left\|p_{n}^{*}(f)-p_{n}^{*}(g)\right\|_{K} \leq K_{n}(f)\|f-g\|_{K}^{1 / 2}
$$

where $K_{n}(f)>0$ is independent of $g$.

We can now give the

Construction of $g$ satisfying (2.3). For a given $z_{0} \in \partial K$, our goal is to construct an entire function $g$ such that for some $\delta>0$ and some sequence of integers $n_{k} \rightarrow \infty$ we have $\left|z_{0}-z\right| \geq \delta$ for every $z \in A_{n_{k}}(g), k=1,2, \ldots$. For this purpose we use some ideas of Lorentz [4].

Set $n_{0}:=0, n_{k+1}=2 n_{k}+1, k=0,1, \ldots$, and $q_{k}(z):=\left(z-z_{0}\right)^{n_{k}} p_{n_{k}}(z)$ $\left(q_{0}(z) \equiv 1\right), k=0,1, \ldots$, where $q_{k} \in P_{2 n_{k}}$ is chosen in such a way that $\left\|q_{k}\right\|_{K}=1$ and 0 is its best approximant out of $P_{n_{k}-1}$ (cf. Lemma 3.3).

Next, let $a_{k} \downarrow 0\left(a_{k} \leq 1\right)$ be such that

$$
\sum_{k=0}^{\infty} a_{k} M_{k}<\infty
$$


where $M_{k}:=\max \left\{\left|q_{k}(z)\right|:|z| \leq k\right\}, k=0,1, \ldots$, and

$$
\sum_{k=s}^{\infty} a_{k}<2 a_{s}, \quad s=0,1, \ldots
$$

We shall introduce another restriction for the $a_{k}$ 's later. Set

$$
g(z):=\sum_{k=0}^{\infty} a_{k} q_{k}(z) .
$$

Then condition (3.3) ensures that $g$ is an entire function.

By Lemma 3.2, there exists a $\delta>0$ such that $q_{k}(z)$ tends to zero uniformly on $B_{\delta}=\left\{z:\left|z-z_{0}\right|<\delta\right\}$ as $k \rightarrow \infty$; that is,

$$
t_{k}:=\sup _{z \in B_{\delta}}\left|q_{k}(z)\right| \rightarrow 0 \quad \text { as } k \rightarrow \infty
$$

Set

$$
g_{s}(z):=\sum_{k=0}^{s} a_{k} q_{k}(z), \quad s=0,1, \ldots,
$$

and note that $g_{s} \in P_{2 n_{s}}$. Since $g_{s+1}-g_{s}=a_{s+1} q_{s+1}$ has zero as its best approximant out of $P_{n_{s+1}-1}=P_{2 n_{s}}$, it follows that $p_{2 n_{s}}^{*}\left(g_{s+1}\right)=g_{s}$. Moreover, by (3.4),

$$
\left\|g-g_{s+1}\right\|_{K} \leq \sum_{k=s+2}^{\infty} a_{k}<2 a_{s+2} .
$$

Applying Lemma 3.4 to $f=g_{s+1}$, we have

$$
\left\|p_{2 n_{s}}^{*}\left(g_{s+1}\right)-p_{2 n_{s}}^{*}(g)\right\|_{K}=\left\|g_{s}-p_{2 n_{s}}^{*}(g)\right\|_{K} \leq K_{2 n_{s}}\left(g_{s+1}\right) \sqrt{2 a_{s+2}}
$$

where $K_{2 n_{s}}\left(g_{s+1}\right) \geq 1$ depends only on $a_{0}, a_{1}, \ldots, a_{s+1}$.

We now make the additional assumption that

$$
K_{2 n_{s}}\left(g_{s+1}\right) \sqrt{a_{s+2}}=o\left(a_{s+1}\right) \quad \text { as } s \rightarrow \infty .
$$

Then using (3.7), (3.8), and (3.9) we get

$$
\begin{aligned}
\left\|g-p_{2 n_{s}}^{*}(g)\right\|_{K} & \geq\left\|g_{s+1}-g_{s}\right\|_{K}-\left\|g-g_{s+1}\right\|_{K}-\left\|g_{s}-p_{2 n_{s}}^{*}(g)\right\|_{K} \\
& \geq a_{s+1}-2 a_{s+2}-K_{2 n_{s}}\left(g_{s+1}\right) \sqrt{2 a_{s+2}} \\
& =a_{s+1}+o\left(a_{s+1}\right) .
\end{aligned}
$$

On the other hand, by (3.7), (3.6), (3.8), and (3.9) we have for $z \in B_{\delta} \cap K$,

$$
\begin{aligned}
\left|g(z)-p_{2 n_{s}}^{*}(g, z)\right| & \leq\left|g(z)-g_{s+1}(z)\right|+\left|g_{s+1}(z)-g_{s}(z)\right|+\left|g_{s}(z)-p_{2 n_{s}}^{*}(g, z)\right| \\
& \leq 2 a_{s+2}+a_{s+1} t_{s+1}+K_{2 n_{s}}\left(g_{s+1}\right) \sqrt{2 a_{s+2}} \\
& =o\left(a_{s+1}\right) .
\end{aligned}
$$

Finally, the last inequality and (3.10) imply that for $s$ large enough, $A_{2 n_{s}}(g) \cap B_{\delta}=$ $\varnothing$.

ACKNOWLEDGMENT. The authors are indebted to H. Stahl for his helpful comments. 


\section{REFERENCES}

1. W. H. Fuchs, On Chebyshev approximation on sets with several components, Proc. NATO Adv. Study Inst., Univ. of Durham, 1979, Academic Press, New York, 1980, pp. 399-408.

2. M. I. Kadec, On the distribution of points of maximal deviation in the approximation of continuous functions by polynomials, Uspekhi Mat. Nauk 15 (1960), 199-202.

3. A. Kro6, On the distribution of points of maximal deviation in complex Čebyšev approximation, Anal. Math. 7 (1981), 257-263.

4. G. G. Lorentz, Distribution of alternation points in uniform polynomial approximation, Proc. Amer. Math. Soc. 92 (1984), 401-403.

5. D. J. Newman and H. S. Shapiro, Some theorems on Cebyšev approximation, Duke Math J. 30 (1963), 673-682.

6. A. Pinkus, $n$-widths in approximation theory, Springer-Verlag, Berlin, 1985.

7. H. S. Shapiro, Topics in approximation theory, Lecture Notes in Math., Vol. 187, SpringerVerlag, Berlin, 1971.

8. Sp. Tashev, On the distribution of the points of maximal deviation for the polynomials of best Chebyshev and Hausdorff approximations, Approximation and Function Spaces (Z. Ciesielski, ed.), North-Holland, Amsterdam, 1981, pp. 791-799.

9. M. Tsuji, Potential theory in modern function theory, 2nd ed., Chelsea, New York, 1958.

10. J. L. Walsh, Interpolation and approximation by rational functions in the complex domain, Amer. Math. Soc. Colloq. Publ., vol. 20, Amer. Math. Soc., Providence, R.I., 1935; 5th ed., 1969.

Mathematical Institute of the Hungarian ACADEmy of SCIENCES, Budapest, REÁlTANODA U. 13-15, H-1053, HUNGARY

institute for Constructive Mathematics, University of SOUth Florida, TAMPA, FLORIDA 33620 